\title{
MULTI-TRAITS OF NON-PATHOGENIC FLUORESCENT PSEUDOMONAS AND EVALUATION OF THEIR POTENTIEL AS BIOCONTROL AGENTS
}

\author{
Mehri Inès, Turki Yousra, Ben Rajeb Asma, Kefacha Sana and Hassen Abdennasser \\ Wastewater Treatment Laboratory, Water Research and Technologies, P.O. Box 273, 8020 Soliman, Tunisia
}

Received 2014-01-09; Revised 2014-01-15; Accepted 2014-04-16

\begin{abstract}
In recent years, certain strains of fluorescent pseudomonads called PGPB have drawn attention due to their abilities to promote plant growth. Therefore, in this investigation, we have explored the adhesive properties, the phytostimulator effects and the biocontrol activities of 40 isolates, with the aim to select potential inoculants to improve crop yields. Ten different colony morphotypes were detected on CRA plates. PsTp172 described as "ST" showed the highest adherence ability to abiotic surface $\left(\mathrm{OD}_{550}=2.102\right) .31$ isolates were positive for the plant growth-promoting hormone (IAA) production and 30 stains solubilised tri-calcium phosphate in Pikovskaya's agar. Furthermore, the highest pyoverdine concentration was detected with PsTp172 strain $(172.50 \mu \mathrm{M})$ under iron starvation conditions. This strain also exhibited a coresistance against $\mathrm{Zn}^{2+}$ and $\mathrm{Mn}^{2+}$ and displayed high values of Minimum Inhibitory Concentrations (MIC) for each heavy metal. Additionally, among the tested isolates, eight strains (PsS15, PsTp172, PsS28, PsTp171, PsS31, PsS67, PsS18, PsS39 and PsS93) were found to be efficient antagonists against the 3 pathogenic strains and 6 isolates (PsS15, PsTp156, PsTp172, PsC54, PsTp171 and PsS102) were considered as lactone inhibitors of the 3 tested strains, as shown by their ability to inhibit the cellular communication. The majority of isolates showed various phytobeneficial traits and the most effective strains are $P$. putida (PsTp172 and PsS15) and P. mosseli (PsTp171).
\end{abstract}

Keywords: Fluorescent Pseudomonads, PGPB, Biofertilizer, Biocontrol, Biofilm

\section{INTRODUCTION}

In diver's environments, beneficial plant-associated bacteria exhibit a significant role in plant growth and health (Wahyudi et al., 2011). The success of Plant Growth Promoting Bacteria (PGPB) is due to their efficient colonization of plant roots by forming microcolonies or biofilms which promotes the plantmicrobe interaction. These bacterial consortiums have the ability to communicate chemically with one another through quorum-sensing (Mishra et al., 2010). This signalling mechanism coordinates bacterial communications with plants, comprising antibiotic production, phytohormone excretion, toxin release and Horizontal Gene Transfer (HGT) (Von Bodman et al., 2003). Though, it has been portrayed that certain PGPB are Corresponding Author: Mehri, I., Wastewater Treatment Laboratory, Water Research and Technologies, P.O. Box 273, 8020 Soliman, Tunisia

able to quench phytopathogens quorum-sensing capability by degrading autoinducer signals, thereby blocking expression of virulence genes (Compant et al., 2005).

An efficient PGPB should display more than two plant growth promoting activities which are root colonization competency, biofertilizer, phytostimulator and phytopathogen biocontrol activities (Bloemberg and Lugtenberg, 2001). Of the various rhizospheric bacteria, Pseudomonas species are the most popular bacteria that combine all these characters; enhance plant growth by direct and indirect mechanisms. The direct promotion involves either supplying plant with growth promoting substances (such as auxins, gibberellins) or helping plant nutrients uptake from environments (Husen et al., 2011). The indirect promotion arises when PGPB avoid harmful effects of phytopathogenic microorganisms (bacteria, 
fungi, nematode) by the production of siderophores, HCN, antibiotics, bacteriocins, volatile metabolites (Saharan and Nehra, 2011).

The performance of PGPB is influenced by a wide range of the environmental conditions (soil composition or characteristics: $\mathrm{pH}$, heavy metals contamination, weather conditions, etc.) that may affect the plant growth (Sajani and Muthukkaruppan, 2011). So, application of highly heavy metal tolerant PGPB has been exploited in contaminated soil allowing the vegetables grow under hard conditions (Zhuang et al., 2007).

Therefore to exploit the potential of native strains of non-pathogenic fluorescent Pseudomonas, the present study attempts to characterize, screen and select nonpathogenic Pseudomonas bacteria exhibiting the highest number of traits.

\section{MATERIALS AND METHODS}

\subsection{Growth media and culture conditions}

All samples were transfered to the laboratory in sterile stomacher containers, stocked at $4{ }^{\circ} \mathrm{C}$ and analyzed within $24 \mathrm{~h} .10 \mathrm{~g}$ or $10 \mathrm{~mL}$ of sample were suspended in $90 \mathrm{~mL}$ sterile distilled water. $50 \mu \mathrm{L}$ of the appropriate dilution was spread on King's B (King et al., 1954) agar medium. After $48 \mathrm{~h}$ of incubation at $25^{\circ} \mathrm{C}$, each different colonie showing fluorescent halo was_purified (Munsch et al., 2000). Each colonie confirmed as putative Pseudomonas spp. (gram negative, catalase positive and oxidase positive) was utilized for further characterization.

The fluorescence was detected in the liquid iron-poor growth medium (Meyer and Abdallah, 1978), the Casamino Acid (CAA) medium, consisting of (per liter) $5 \mathrm{~g}$ of low-iron Bacto Casamino Acid (Difco), $1.54 \mathrm{~g}$ of $\mathrm{K}_{2} \mathrm{HPO}_{4} \cdot 3 \mathrm{H}_{2} \mathrm{O}$ and $0.25 \mathrm{~g}$ of $\mathrm{MgSO}_{4} \cdot 7 \mathrm{H}_{2} \mathrm{O}$.

Strains whose designations begin with PsS, PsTp, $\mathrm{PsC}$ and $\mathrm{PsWw}$ were collected respectively from soil rhizosphere, wastewater treatment plant rhizosphere, compost and wastewater.

The NCBI Accession Numbers for the 16S rDNA gene sequences of 40 isolates determined in this present investigation are listed in Table 1.

\subsection{Adhesive Properties}

\subsubsection{Qualitative Detection of Biofilm Formation (Detection of Colony Morphology Variant)}

All strains were spread on Congo Red Agar (CRA) containig $0.8 \mathrm{~g}$ of Congo red (Sigma) and $36 \mathrm{~g}$ of saccharose to 11 of brain heart infusion agar (Bio-rad). A concentrated aqueous solution of Congo red stain was prepared, autoclaved separately for $15 \mathrm{~min}$ at $121^{\circ} \mathrm{C}$ and added when the agar had cooled to $55^{\circ} \mathrm{C}$ (Freeman et al., 1989). Plates were incubated at $30^{\circ} \mathrm{C}$ for $48 \mathrm{~h}$. Morphology, pigmentation and mucosity were observed.

\subsection{Quantitative Estimation of Biofilm Formation on Polystyrene Surface}

All bacteria were cultured overnight in Brain Infusion Broth (BHI-0.25 glucose at $30^{\circ} \mathrm{C}$ ). The culture was diluted 1:20 in fresh BHI plus $(0.25 \%)$ glucose at $30^{\circ} \mathrm{C}$. This suspension $(200 \mu \mathrm{L})$ was utilized to inoculate sterile 96-well-polystyrene microtiter plates. The plates were incubated at $30^{\circ} \mathrm{C}$ aerobically for $24 \mathrm{~h}$. The cultures were eliminated and the microtiter wells were washed twice with phosphate-buffered saline (7 $\mathrm{mM} \mathrm{Na} \mathrm{HPO}_{4}, 3 \mathrm{mM} \mathrm{NaH} \mathrm{PO}_{4}$ and $130 \mathrm{mM} \mathrm{NaCl}$ at $\mathrm{pH}$ 7.4) to remove non-adherent cells and were dried in an inverted position. Then, bacteria that not adherate to microtiter plates were stained with $1 \%$ Crystal violet for $15 \mathrm{~min}$. The wells were washed once more and the Crystal violet was dissolved in $200 \mu \mathrm{L}$ of ethanol (95\%). An automated PR3 100 TSC (Bio-Rad) was used to measure the absorbance at $550 \mathrm{~nm}\left(\mathrm{OD}_{550}\right)$ (O'Toole et al., 1999). Each essay was performed in triplicate. The following values were attributed for biofilm determination: $\mathrm{OD}_{550} \leq 0.1$ non biofilm forming; $0.1 \leq \mathrm{OD}_{550} \leq 0.5$ weak biofilm forming; $0.5 \leq \mathrm{OD}_{550} \leq 1$ medium biofilm forming; $1 \leq \mathrm{OD}_{550} \leq 2$ strong biofilm forming; $\mathrm{OD}_{550} \geq 2$ very strong biofilm forming.

\subsection{Plant Growth Promoter Activity 2.4.1. Indole-3-Acetic Acid (IAA) Production}

The production of Indole-3-Acetic Acid (IAA) by the 40 isolates of Pseudomonas was assayed following the technique portrayed by Patten and Glick (2002). The auxin amount was determined spectrophotometrically after $72 \mathrm{~h}$ of incubation at $535 \mathrm{~nm}$ in minimal medium containing (per liter) $\mathrm{MgSO}_{4} \cdot 7 \mathrm{H}_{2} \mathrm{O}, 0.2 \mathrm{~g} ; \mathrm{KH}_{2} \mathrm{PO}_{4}, 6.8$ g; $\left(\mathrm{NH}_{4}\right)_{2} \mathrm{SO}_{4}, 2.0 \mathrm{~g}$; citrate, $2.0 \mathrm{~g}$; $\mathrm{ZnO}, 0.006 \mathrm{~g}$; $\mathrm{H}_{3} \mathrm{BO}_{3}, 0.006 \mathrm{~g} ; \mathrm{FeCl}_{3} \cdot 6 \mathrm{H}_{2} \mathrm{O}, 0.0024 \mathrm{~g} ; \mathrm{CaCO}_{3}, 0.02 \mathrm{~g}$ and $\mathrm{HCl}, 0.13 \mathrm{~mL}$, supplemented with glucose $(10 \mathrm{~g})$ and L-tryptophan $\left(100 \mu \mathrm{g} \mathrm{mL} \mathrm{m}^{-1}\right)$, using Salkowski's reagent (Gordon and Weber, 1951). In each culture medium, the IAA concentration was established by comparison with a standard curve.

\subsection{Phosphate Solubilisation}

Onto Pikovskaya's agar medium containing (per liter): $10 \mathrm{~g}$ dextrose; $0.5 \mathrm{~g}$ yeast extract; $5 \mathrm{~g} \mathrm{Ca}_{3}\left(\mathrm{PO}_{4}\right)_{2}$; $0.5 \mathrm{~g}\left(\mathrm{NH}_{4}\right)_{2} \mathrm{SO}_{4} ; 0.2 \mathrm{~g} \mathrm{KCl} ; 0.1 \mathrm{~g} \mathrm{MgSO}_{4} \cdot 7 \mathrm{H}_{2} \mathrm{O} ; 0.0001 \mathrm{~g}$ 
$\mathrm{FeSO}_{4} \cdot 7 \mathrm{H}_{2} \mathrm{O} ; 0.0001 \mathrm{~g} \mathrm{MnSO}_{4} \cdot \mathrm{H}_{2} \mathrm{O}$ and $15 \mathrm{~g}$ agar, all cells were streaked. After $72 \mathrm{~h}$ of incubation at $28^{\circ} \mathrm{C}$, strains inducing clear zone around colonies were noted as positive (Katznelson and Bose, 1959).

\subsection{Biocontrol Activity 2.6.1. Pyoverdine Assay}

The method was described in details in previous study (Mehri et al., 2012). Pyoverdin levels were calculated using the extinction coefficient $\left(\varepsilon=20000 \mathrm{M}^{-1} \mathrm{~cm}^{-1}\right)$ following the thechnique of Meyer and Abdallah (1978). Three repeated experiments were envisaged.

\subsection{Heavy Metal Tolerance}

Metal Salts were utilized in the following forms: $\mathrm{ZnSO}_{4} \cdot 7 \mathrm{H}_{2} \mathrm{O}$ (iron content, $<10 \mathrm{ppm}$ ) and $\mathrm{MnSO}_{4} \cdot \mathrm{H}_{2} \mathrm{O}$ (iron content, $<0.001 \%$ ). Stocks of $10 \mathrm{mM}^{1} \mathrm{ZnSO}_{4}$ and $\mathrm{MnSO}_{4}$ salts were prepared and sterilized with $0.22 \mu \mathrm{m}$ filters under aseptic condition. The stock solutions were incorporated to autoclaved CAA media (CAA+Mn and $\mathrm{CAA}+\mathrm{Zn})$ at a final concentration of $60 \mu \mathrm{M}$ for each metal. In the purpose to establish the Maximum Tolerable Concentration (MTC) of metals at which growth was observed, the 40 strains were monitored in CAA medium as a function of increasing concentrations of $\mathrm{Mn}_{-}$(II) and $\mathrm{Zn}$ (II) from 0.1 to $475 \mu \mathrm{M}$ in $5 \mathrm{~mL}$ CAA medium (Mehri et al., 2012).

\subsection{In Vitro Antagonism Against Pathogenic and Phytopathogenic Pseudomonas Bacteria}

Culture supernatant was prepared as follows: A centrifugation at $150 \mathrm{rpm}$ of an overnight culture. Resulting supernatant was neutralized, sterilized by filtering and assayed for the presence of an inhibitor in the broth; following the agar well diffusion assay technique (Barefoot et al., 1983). Nutrient agar was first seeded with pathogenic organism $(110 \mu \mathrm{L}$ of overnight culture per $20 \mathrm{~mL}$ of agar) in sterile Petri dishes and after solidification, dried for $15 \mathrm{~min}$. Wells were bored in the agar. Aliquots of the supernatant were distributed in holes and plates were incubated $24 \mathrm{~h}$ at $30^{\circ} \mathrm{C}$. Inhibition of growth was determined by an area of inhibition surrounding each agar well.

\subsection{Quorum-Quenching (QQ) Bioassays}

N-Acyl Homoserine Lactone (NAHL) degradation ability of the isolates was detected by cross streaking against Chromobacterium violaceum CV026 as the AHL biosensor (Swift et al., 1999). Supernatants from 7 days old cultures of the isolates were adjusted to $\mathrm{pH} 7$ and
$15 \mu 1$ of each supernatant blended with pathogenic or phytopathogenic Pseudomonas supernatant and dispensed in the wells of a bioassay plate overlaid with C. violaceum CV026.

\subsection{Pathogens and Phytopathogens Used in Antagonistic and QQ Assays}

3 strains of Pseudomonas were utilized in this study: $P$. aeruginosa (PsCLHMC1) from clinical origin, P. Otitidis (PsWw118) isolated from wastewater (Mehri et al., 2011) and P. savastanoi from olive knots (Ouzari et al., 2008). Access numbers of Pseudomonas strains were respectively: HM627572, HM627606 and AM265392.

\section{RESULTS}

The 40 isolates were screened for functional properties. PGPB traits were represented in Table 1.

\subsection{Growth Temperature Tolerance}

All isolates, except three strains, were able to grow at maximum temperature of $32^{\circ} \mathrm{C}$. On the other hand PsS60, PsS91 and PsS73 strains were able to grow at $37^{\circ} \mathrm{C}$ and $42^{\circ} \mathrm{C}$.

\subsection{Colony Morphology on CRA Plates}

The studied isolates showed ten different colony morphotypes on CRA plates_(Fig. 1). The morphotype "ST", described in previous study (Kirisits et al., 2005), was detected in the strain PsTp172 showing rough, wrinkled and sticky colony (Fig. 1a). Another morphotype "SM", previously described by Rainey and Travisano (1998), was found in the strain PsS2 appearing as smooth and regular colony (Fig. 1b). Colony morphotype of PsS103 and PsTp169 isolates were light rose or colourless (Fig. 1c) suggesting that biofilm formation was affected (Rakhimova et al., 2008). Biofilm growth of PsTp155 produced glossy colonies with an inner circle of lysed cells (Fig. 1d) (Rakhimova et al., 2008). PsS4 and PsS46 showed a particular morphotypes characterized, respectively, by a flat colony having orange pigment in the center of the colony (Fig. 1e) and a translucent colony (Fig. 1f). The remaining strains exhibited non mucoïd ( 2 isolates, Fig. 1g), mucoïd (19 isolates, Fig. 1h), highly mucoïd with the dye Congo red concentrated in colony center (11 isolates, Fig. 1i) and stronger mucoïd (one isolate, Fig.1j) structures. In fact, the mucosity indicates a weak or a high production of exopolysaccharide (alginate) (Kirisits et al., 2005). 

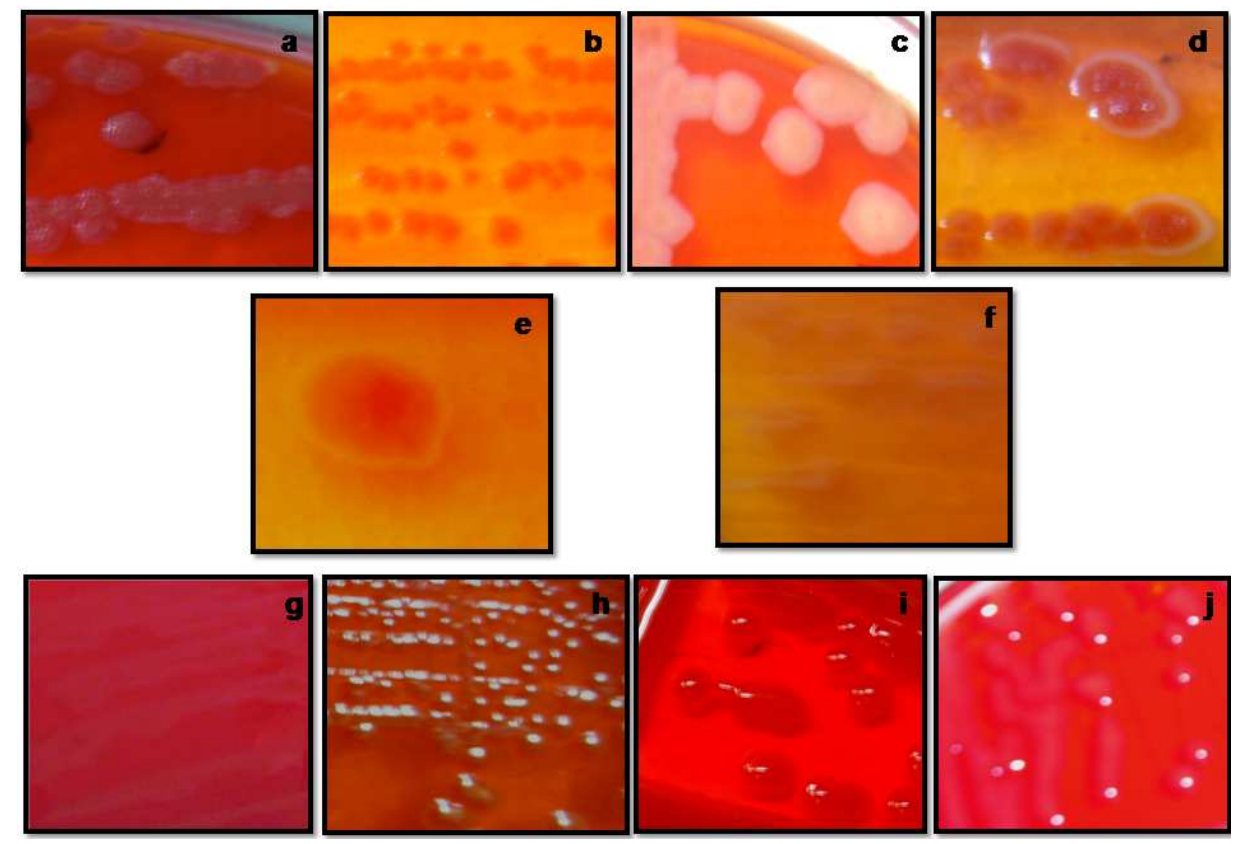

Fig. 1. Colony morphology variants on Congo Red Agar (CRA): (a) rough, wrinkled and sticky "ST"; (b) smooth and regular "SM" (c) light rose or colourless (d) glossy with an inner circle of lysed cells (e) flat with orange pigment in the center (f) translucent (g) non mucoïd (h) mucoïd (i) highly mucoïd with the dye Congo red concentrated in colony center (j) stronger mucoïd

\subsection{Biofilm Formation on Polystyrene Surface}

About 26 isolates showing mucoid, highly mucoïd and stronger mucoïd colony morphotypes were nonbiofilm forming on polystyrene surfaces with an $\mathrm{OD}_{550} \leq 0.1$. While, PsTp172 (morphotype "ST") produced a very large amount of biofilm $\left(\mathrm{OD}_{550}=\right.$ 2.102). This type of morphotype was characterized by hyper-adhesion and autoaggregation to abiotic surfaces (Kirisits et al., 2005). The PsS67 strain was strongly adhesive to polystyrene with a value of 1,238 at $550 \mathrm{~nm}$. Strains PsTp171 and PsS46 were medium adhesive to the abiotic surface with $\mathrm{OD}_{550}$ values respectively of 0.639 and 0.547. On the other hand, Rakhimova et al. (2008) explained that isolates with light rose or colourless colony morphotypes, suggesting that biofilm formation was affected. Hence PsTp169 and PsS103 showed optical densities respectively of 0.069 and 0.041. All these data were summarized in Table 1.

\subsection{IAA production and Phosphorus solubilisation}

About 31 strains (76\%) of fluorescent Pseudomonas produced the IAA hormone in various levels, while 9 strains showed no producing activity. The isolate PsS93 produced the highest level of IAA $\left(7.4 \mu \mathrm{g} \mathrm{mL} \mathrm{mL}^{-1}\right.$ ), whereas PsS4 and PsS31 were found the weak producer isolates (respectively $0.11-0.15 \mu \mathrm{g} \mathrm{mL}^{-1}$ ). On the other hand, 30 Pseudomonas spp. $(75 \%)$ were capable to solubilise, in Pikovskaya's agar, tri-calcium phosphate by the formation of clear halo zone (Fig. 2).

\subsection{Pyoverdine Quantification}

When the cells grown under iron starvation Conditions (CAA medium) different level of siderophore was detected in culture supernatant. The highest metabolite concentration was obtained with PsTp172 strain $(172.50 \mu \mathrm{M})$. The least pyoverdine production was detected in PsS73 isolate $(2.82 \mu \mathrm{M})$.

\subsection{Heavy metals Resistance}

The 40 isolates were screened for their metal tolerance on CAA media containing different metal concentrations of each $\mathrm{Zn}^{2+}$ and $\mathrm{Mn}^{2+}$. The MTC for each metal was determined to select isolates able to grow and resist high level of metal toxicity. Generally, the bacterial isolates showed the maximum resistance against $\mathrm{Zn}^{2+}$ (MTC: 100-300 $\mu \mathrm{M}$ ) and $\mathrm{Mn}^{2+}$ (MTC: $300-475 \mu \mathrm{M})$. PsTp172 strain was the most tolerant 
bacteria to tested metals. This isolate resisted to $\mathrm{Zn}^{2+}$ up to $300 \mu \mathrm{M}$ and to $\mathrm{Mn}^{2+}$ up to $475 \mu \mathrm{M}$.

\subsection{Antagonism Assay Against Pathogenic and Phytopathogenic Pseudomonas}

27 isolates inhibited the growth of at least one pathogenic strain. PsS15, PsTp172, PsS28, PsTp171, PsS31, PsS67, PsS18, PsS39 and PsS93 were found to be efficient antagonists against the 3 tested strains (Fig. 3).

\subsection{Quorum-Quenching Activity}

The C. Violaceum CV026 was used as an indicator of extracellular N-Acyl Homoserine Lactone (NAHL) inhibition of $P$. aeruginosa, P.otitidis and P. savastanoi; 18 isolates were considered as lactone inhibitors as shown by their ability to inhibit violacein production of at least one pathogenic strain. PsS15, PsTp156, PsTp172, PsC54, PsTp171 and PsS102 were found to inhibit the cellular communication factor of the 3 tested strains (Fig. 4).
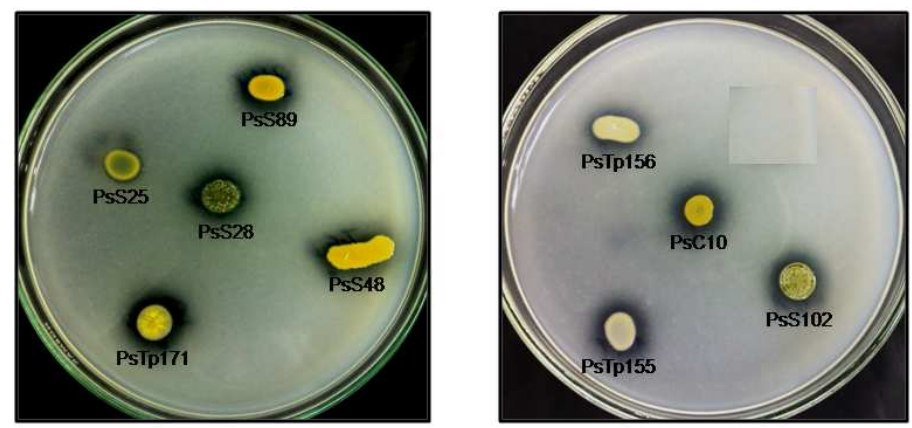

Fig. 2. Phosphate solubilisation halo produced by Pseudomonas isolates


Fig. 3. Antagonistic effect of Pseudomonas isolates on pathogenic Pseudomonas (P. savastanoi and P. otitidis)

P. savastanoi

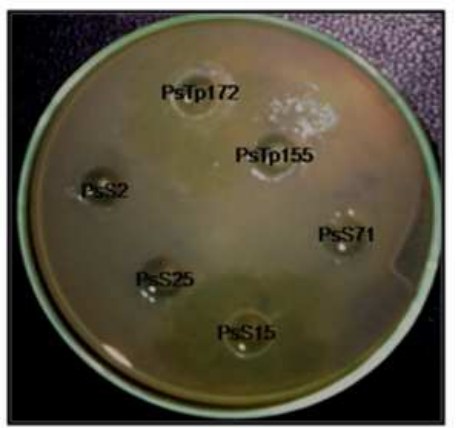

P. otitisid

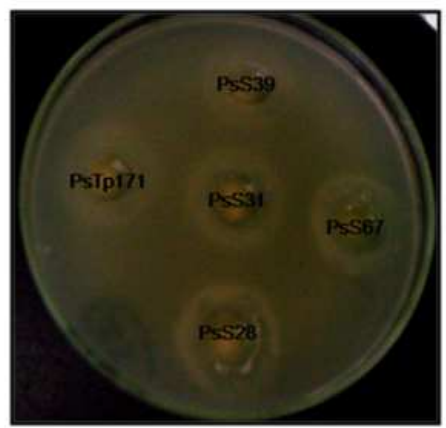

Fig. 4. NAHL inhibition activity (QQ) of PsS15 and PsC54 via P. aeruginosa (P. a) and P. savastanoi (P. s) 
Mehri Inès et al. / American Journal of Environmental Science 10 (2): 199-209, 2014

Table 1. List of the forty fluorescent Pseudomonas isolates and their PGPB traits and biocontrol activities

\begin{tabular}{|c|c|c|c|c|c|c|c|c|c|c|c|c|c|c|c|c|}
\hline \multirow[b]{2}{*}{ Strains } & \multirow{2}{*}{$\begin{array}{l}\text { Closest16S } \\
\text { r DNA speice } \\
\text { (assession } \\
\text { Number) }\end{array}$} & \multirow{2}{*}{$\begin{array}{l}\text { Growth } \\
\text { at } 37 \\
-42^{\circ} \mathrm{C}\end{array}$} & \multirow{2}{*}{$\begin{array}{l}\text { Biofilm } \\
\text { form } \\
\text { ation } \\
\left(\mathrm{OD}_{550}\right)\end{array}$} & \multirow[b]{2}{*}{ Morphotypes $^{\mathrm{a}}$} & \multirow{2}{*}{$\begin{array}{l}\text { IAA } \\
\text { produ } \\
\text { ction } \\
(\mu \mathrm{g} \mathrm{mL})\end{array}$} & \multirow{2}{*}{\multicolumn{2}{|c|}{$\begin{array}{l}\text { Phos } \\
\text { phate } \\
\text { solubiliz } \\
\text { ationa }\end{array}$}} & \multicolumn{3}{|c|}{$\begin{array}{l}\text { Antagonistic } \\
\text { ctivity }^{\mathrm{b}}\end{array}$} & \multicolumn{3}{|c|}{$\begin{array}{l}\text { NAHL } \\
\text { inhabition }(\mathrm{QQ})^{\mathrm{c}}\end{array}$} & \multirow{2}{*}{$\begin{array}{l}\text { Sidero } \\
\text { phore } \\
\text { produc } \\
\text { tion }(\mu \mathrm{M})\end{array}$} & \multirow{2}{*}{$\begin{array}{l}\text { MTC } \\
(\mu \mathrm{M} \\
\text { of } \\
\mathrm{Zn}) \\
\end{array}$} & \multirow{2}{*}{$\begin{array}{l}\text { MTC } \\
(\mu \mathrm{M} \\
\text { of } \\
\text { Mn })\end{array}$} \\
\hline & & & & & & & & p.a & p.o & p.s & p.a & p.o & p.s & & & \\
\hline PsS2 & $\begin{array}{l}\text { P. Putida } \\
\text { (HM627611) }\end{array}$ & - & 0,266 & $\begin{array}{l}\text { smooth morph } \\
\text { :'SM" }\end{array}$ & 0,27 & ++ & & - & - & - & - & - & - & 35,33 & 100 & 350 \\
\hline PsS4 & $\begin{array}{l}\text { Pseubom on sp } \\
\text { (HM627612) }\end{array}$ & - & 0,135 & $\begin{array}{l}\text { Flat, or pigment } \\
\text { incolony center }\end{array}$ & 0,11 & + & & - & - & - & - & - & - & 14,90 & 150 & 350 \\
\hline PsS15 & $\begin{array}{l}\text { P. Putida } \\
\text { (HM627595) }\end{array}$ & - & 0,021 & Mucoid & 1,50 & + & & + & + & + & + & + & + & 32,83 & 200 & 400 \\
\hline PsS46 & $\begin{array}{l}\text { P. Putida } \\
\text { (HM627613) }\end{array}$ & - & 0,547 & Flat translucent & 2,52 & + & & - & - & + & - & - & + & 98,18 & 250 & 450 \\
\hline PsWw124 & $\begin{array}{l}\text { P. Putida } \\
\text { (HM 627628) }\end{array}$ & - & 0,007 & Mucoid & 1,25 & + & & - & + & + & - & - & + & 18,17 & 150 & 300 \\
\hline PsTp169 & $\begin{array}{l}\text { P. Putida } \\
\text { (HM 627607) }\end{array}$ & - & 0,040 & Mucoid & 1,35 & + & & + & - & + & + & + & + & 13,87 & 200 & 300 \\
\hline PsTp 172 & $\begin{array}{l}\text { P. Putida } \\
\text { (HM 627615) }\end{array}$ & - & 0,041 & $\begin{array}{l}\text { Flat, light ro } \\
\text { colourless }\end{array}$ & - & + & & + & + & - & + & + & + & 106,17 & 250 & 450 \\
\hline PsTp 172 & $\begin{array}{l}\text { P. Putida } \\
\text { (HM 627615) }\end{array}$ & - & 2,102 & $\begin{array}{l}\text { Rough, wrinkled, } \\
\text { sticky: "ST" }\end{array}$ & 0,27 & + & & + & + & + & + & + & + & 172,50 & 300 & 475 \\
\hline PsS 28 & $\begin{array}{l}\text { P. Putida } \\
\text { (HM 627615) }\end{array}$ & - & 0,176 & Mucoid & - & ++ & & + & + & + & + & + & - & 85,33 & 200 & 400 \\
\hline PsC 54 & $\begin{array}{l}\text { P. Putida } \\
\text { (HM 627615) }\end{array}$ & - & 0,103 & Mucoid & 1,45 & + & & - & + & + & + & + & + & 49,62 & 200 & 350 \\
\hline PsS 103 & $\begin{array}{l}\text { P. Putida } \\
\text { (HM 627615) }\end{array}$ & - & 0,069 & $\begin{array}{l}\text { Flat, light ro } \\
\text { colourless }\end{array}$ & 1,02 & + & & + & - & - & + & - & - & 80,80 & 200 & 300 \\
\hline PsS 48 & $\begin{array}{l}\text { P. Putida } \\
\text { (HM 627615) }\end{array}$ & - & 0,032 & Mucoid & 0,78 & ++ & & - & - & - & - & + & - & 31,50 & 150 & 350 \\
\hline PsS 79 & $\begin{array}{l}\text { P. Putida } \\
\text { (HM 627615) }\end{array}$ & - & 0,042 & Mucoid & - & + & & - & - & - & + & - & - & 31,50 & 150 & 300 \\
\hline PsS 71 & $\begin{array}{l}\text { P. Putida } \\
\text { (HM 627615) }\end{array}$ & - & 0,095 & Mucoid & 1,41 & ++ & & - & - & - & - & - & - & 85,38 & 250 & 350 \\
\hline PsS 11 & $\begin{array}{l}\text { P.m. onteilii } \\
\text { (HM 627615) }\end{array}$ & - & 0,426 & Mucoid & 0,82 & + & & + & - & - & + & + & + & 65,90 & 200 & 400 \\
\hline PsTp 139 & $\begin{array}{l}\text { P. Putida } \\
\text { (HM 627615) }\end{array}$ & - & 0,007 & Mucoid & 1,11 & ++ & & - & - & - & - & - & - & 52,50 & 200 & 350 \\
\hline PsTp 153 & $\begin{array}{l}\text { P. Putida } \\
\text { (HM 627615) }\end{array}$ & - & 0,003 & Mucoid & 1,21 & + & & - & - & - & - & - & - & 12,10 & 100 & 300 \\
\hline PsTp 155 & $\begin{array}{l}\text { P.m. onteilii } \\
\text { (HM 627615) }\end{array}$ & - & 0,405 & $\begin{array}{l}\text { Shiny autolysis } \\
\text { (inner circle of } \\
\text { Lysed cell) }\end{array}$ & 0,91 & ++ & & - & - & - & - & - & - & 17,22 & 150 & 300 \\
\hline PsTp 154 & $\begin{array}{l}\text { P.m. onteilii } \\
\text { (HM 627615) }\end{array}$ & - & 0,450 & Mucoid & 0,98 & + & & - & - & - & - & - & - & 11,55 & 100 & 300 \\
\hline PsC 10 & $\begin{array}{l}\text { P. Putida } \\
\text { (HM 627615) }\end{array}$ & - & 0,156 & Mucoid & 0,62 & ++ & & + & + & - & & $\mathrm{b}$ & & 21,33 & 150 & 400 \\
\hline PsC 75 & $\begin{array}{l}\text { P. Putida } \\
\text { (HM 627615) }\end{array}$ & - & 0,008 & Mucoid & - & + & & + & + & - & + & + & - & 17,67 & 100 & 300 \\
\hline PsT p171 & $\begin{array}{l}\text { P.mosseli } \\
\text { (HM627603) }\end{array}$ & & 0,639 & lat,non m ucoid & - & +++ & + & + & + & + & + & + & & 82,35 & 200 & 425 \\
\hline PsS31 & $\begin{array}{l}\text { P.Putida } \\
\text { (HM 627624) }\end{array}$ & & 0,017 & nucoid & 0,15 & ++ & + & + & + & & $\mathrm{b}$ & & & 21,23 & 100 & 300 \\
\hline PsS102 & (HM 627601) & & 0,081 & nucoid & + & ++ & + & + & + & + & + & + & & 34,76 & 100 & 400 \\
\hline
\end{tabular}


Mehri Inès et al. / American Journal of Environmental Science 10 (2): 199-209, 2014

Table 1. Continue

\begin{tabular}{|c|c|c|c|c|c|c|c|c|c|c|c|c|c|c|c|}
\hline \multirow[b]{2}{*}{ Strains } & \multirow{2}{*}{$\begin{array}{l}\text { Closest16S } \\
\text { r DNA speice } \\
\text { (assession } \\
\text { Number) }\end{array}$} & \multirow{2}{*}{$\begin{array}{l}\text { Growth } \\
\text { at } 37 \\
-42^{\circ} \mathrm{C}\end{array}$} & \multirow{2}{*}{$\begin{array}{l}\text { Biofilm } \\
\text { form } \\
\text { ation } \\
\left(\mathrm{OD}_{550}\right)\end{array}$} & \multirow{2}{*}{ Morphotypes $^{\mathrm{a}}$} & \multirow{2}{*}{$\begin{array}{l}\text { IAA } \\
\text { produ } \\
\text { ction } \\
(\mu \mathrm{g} \mathrm{mL})\end{array}$} & \multirow{2}{*}{$\begin{array}{l}\text { Phos } \\
\text { phate } \\
\text { solubiliz } \\
\text { ationa }\end{array}$} & \multicolumn{3}{|c|}{$\begin{array}{l}\text { Antagonistic } \\
\text { ctivity }^{\mathrm{b}}\end{array}$} & \multicolumn{3}{|c|}{$\begin{array}{l}\text { NAHL } \\
\text { inhabition }(\mathrm{QQ})^{\mathrm{c}}\end{array}$} & \multirow{2}{*}{$\begin{array}{l}\text { Sidero } \\
\text { phore } \\
\text { produc } \\
\text { tion }(\mu \mathrm{M}) \\
\end{array}$} & \multirow{2}{*}{$\begin{array}{l}\text { MTC } \\
(\mu \mathrm{M} \\
\text { of } \\
\text { Zn }) \\
\end{array}$} & \multirow{2}{*}{$\begin{array}{l}\text { MTC } \\
(\mu \mathrm{M} \\
\text { of } \\
\text { Mn })\end{array}$} \\
\hline & & & & & & & p.a & p.o & p.s & p.a & p.o & p.s & & & \\
\hline PsS67 & $\begin{array}{l}\text { P.Putida } \\
\text { (HM 627602) }\end{array}$ & - & 1,238 & $\begin{array}{l}\text { flat,non mucoid } \\
\text { mucoid }\end{array}$ & - & + & + & - & + & & $\mathrm{b}$ & & 8567 & 250 & 425 \\
\hline PsS18 & $\begin{array}{l}\text { P.Putida } \\
\text { (HM 627510) }\end{array}$ & - & 0,001 & $\begin{array}{l}\text { stronger mucoid } \\
\text { (moist and bubbly) }\end{array}$ & 0,82 & + & + & + & + & & $\mathrm{b}$ & & 3567 & 150 & 350 \\
\hline PsWw128 & $\begin{array}{l}\text { P.fluore scens } \\
\text { (HM 627582) }\end{array}$ & - & 0,053 & mucoid & 4,83 & ++ & + & + & + & - & - & - & 24,55 & 100 & 300 \\
\hline PsS89 & $\begin{array}{l}\text { P.fluore scens } \\
\text { (HM 627583) }\end{array}$ & - & 0,139 & $\begin{array}{l}\text { Highly mucoid dye } \\
\text { Congo red in the } \\
\text { center of colony }\end{array}$ & 1,53 & ++ & - & - & - & - & - & + & 77,67 & 200 & 425 \\
\hline PsS60 & $\begin{array}{l}\text { P.fluore scens } \\
\text { (HM 627584) }\end{array}$ & + & 0,031 & $\begin{array}{l}\text { Highly mucoid dye } \\
\text { Congo red in the } \\
\text { center of colony }\end{array}$ & 1,30 & - & - & - & - & & $\mathrm{b}$ & & 5,17 & 250 & 425 \\
\hline PsS29 & $\begin{array}{l}\text { P.fluorescens } \\
\text { (HM 627585) }\end{array}$ & & 0,010 & $\begin{array}{l}\text { Highly mucoid dye } \\
\text { Congo red in the } \\
\text { center of colony }\end{array}$ & 1,18 & - & + & + & - & & $\mathrm{b}$ & & 26,28 & 200 & 475 \\
\hline PsS39 & $\begin{array}{l}\text { P.fluore scens } \\
\text { (HM 627586) }\end{array}$ & - & 0,063 & $\begin{array}{l}\text { Highly mucoid dye } \\
\text { Congo red in the } \\
\text { center of colony }\end{array}$ & 0,03 & - & - & - & + & & $\mathrm{b}$ & & 9,93 & 200 & 450 \\
\hline PsS25 & $\begin{array}{l}\text { P.fluore scens } \\
\text { (HM 627587) }\end{array}$ & - & 0,000 & $\begin{array}{l}\text { Highly mucoid dye } \\
\text { Congo red in the } \\
\text { center of colony }\end{array}$ & 4,49 & $+/-$ & + & + & + & & $\mathrm{b}$ & & 7,37 & 150 & 350 \\
\hline PsS49 & $\begin{array}{l}\text { P.fluore scens } \\
\text { (HM 627588) }\end{array}$ & - & 0,003 & $\begin{array}{l}\text { Highly mucoid dye } \\
\text { Congo red in the } \\
\text { center of colony }\end{array}$ & 7,4 & - & - & - & - & & $\mathrm{b}$ & & 9,07 & 250 & 475 \\
\hline PsS93 & $\begin{array}{l}\text { P.fluore scens } \\
\text { (HM 627589) }\end{array}$ & - & 0,007 & $\begin{array}{l}\text { Highly mucoid dye } \\
\text { Congo red in the } \\
\text { center of colony }\end{array}$ & 4,53 & - & - & - & + & & $\mathrm{b}$ & & 9,85 & 250 & 450 \\
\hline PsS90 & $\begin{array}{l}\text { P.fluore scens } \\
\text { (HM 627590) }\end{array}$ & - & 0,004 & $\begin{array}{l}\text { Highly mucoid dye } \\
\text { Congo red in the } \\
\text { center of colony }\end{array}$ & 3,94 & - & + & + & + & & $\mathrm{b}$ & & 7,15 & 100 & 350 \\
\hline PsS91 & $\begin{array}{l}\text { P.fluore scens } \\
\text { (HM 627591) }\end{array}$ & + & 0,004 & $\begin{array}{l}\text { Highly mucoid dye } \\
\text { Congo red in the } \\
\text { center of colony }\end{array}$ & 2,32 & - & - & - & - & & $\mathrm{b}$ & & 4,12 & 150 & 400 \\
\hline PsS23 & $\begin{array}{l}\text { P.fluore scens } \\
\text { (HM 627592) }\end{array}$ & - & 0,020 & $\begin{array}{l}\text { Highly mucoid dye } \\
\text { Congo red in the } \\
\text { center of colony }\end{array}$ & 2,24 & - & + & - & + & & $\mathrm{b}$ & & 5,32 & 100 & 300 \\
\hline PsS26 & $\begin{array}{l}\text { P.fluore scens } \\
\text { (HM 627593) }\end{array}$ & - & 0,089 & $\begin{array}{l}\text { Highly mucoid dye } \\
\text { Congo red in the } \\
\text { center of colony }\end{array}$ & 2,83 & - & + & - & + & & $\mathrm{b}$ & & 13,62 & 200 & 425 \\
\hline PsS73 & $\begin{array}{l}\text { P.fluore scens } \\
\text { (HM 627594) }\end{array}$ & + & 0,005 & $\begin{array}{l}\text { Highly mucoid dye } \\
\text { Congo red in the } \\
\text { center of colony }\end{array}$ & & - & + & + & - & & $\mathrm{b}$ & & 2,82 & 250 & 475 \\
\hline
\end{tabular}

${ }^{\mathrm{a}}$ The different colony morphotypes after $48 \mathrm{~h}$ incubation at $30^{\circ} \mathrm{C}$ in Congo red agar (Fig. 1.),

${ }^{\mathrm{b}}$ Lactone inhibition activity was tested only with not lactone producers strains (PsC10, PsS31, PsS67, PsS18, PsS60, PsS29, PsS39, PsS25, PsS49, PsS93, PsS90, PsS91, PsS23, PsS26 and PsS73 are lactone producers strains).

IAA: Indole-3-Acetic Acid, NAHL: N-acyl homoserine lactone, QQ: Quorum-Quenching, P. a: Pseudomonas aeruginosa, P. o: Pseudomonas otitidis, P. s: Pseudomonas savastanoi, MTC: Maximum Tolerable Concentration, Zn: Zinc, Mn: Manganese. 


\section{DISCUSSION}

Among PGPB, fluorescent pseudomonads have proved a boon in sustainable agriculture for use as biofertilizer (plant growth promoting capacity), biocontrol agent (antagonistic activity) and in bioremediation (degradation of pollutants) (Husen et al., 2011). A total of 40 isolates recovered from diver's origin were subsequently screened for plant growth promoting characteristics and biological control of plant deleterious microbes.

IAA, a member of phytohormone group, acts as important signal molecule in the regulation of plant growth (Kumar et al., 2012). In our study most of the Pseudomonas isolates were positive for IAA production (76\%). Among them, P. fluorescens strains isolated from soil rhizosphere are found to be good producers of the phytohormone (PsS93, PsS89, PsS49, PsS90). Saharan and Nehra (2011) study revealed that isolates of the Pseudomonas putida-fluorescens group, which produce auxin, are able to induce statistically important yield increases up to $144 \%$ in field tests.

In the soil, most of phosphorus is present in the insoluble form and cannot be used by plants (Pradhan and Sukla, 2006). The study of Rodriguez and Fraga (1999) revealed that the availability of phosphor in soil was increased by strains of Bacillus, Pseudomonas and Rhizobium. In our experiments, all $P$. putida isolates are able to produce the phosphatase enzyme. Villegas and Fortin (2002) selected $P$. putida strains as effective agents able to solubilise soil phosphate. The aptitude of various strains to transform the insoluble phosphate suggested their application in agriculture (Wahyudi et al., 2011).

Apart the PGPB biofertilizer actions, it is prominent to note that the conformation of adherent microbial population affects significantly the duration and intensity of the plant-microbe interactions (Danhorn and Fuqua, 2007). In general, there should be a correlation between the colony morphotype and the capacity of adhesion to different surfaces. Therefore, PsTp172 strain with rough, wrinkled and Sticky (ST) colony morphotype showed a higher adherence ability $\left(\mathrm{OD}_{550}=2.102\right)$. "ST" variant is so called because of the hyperadherence to abiotic surfaces and the strong autoaggregative phenotype in liquid culture (Kirisits et al., 2005). On the other hand, some isolated strains showed hypermucoid colony (PsWw128, PsS60, PsS29). In this context, Bianciotto et al. (2001) described that the hypermucoid mutant of $P$. fluorescens CHA0 demonstrated an important adherence_to roots and to the mycelium of arbuscular mycorrhizal fungi. Exopolysaccharide production (alginate) contributes to surface colonising of plants (Danhorn and Fuqua, 2007). Study of Stoodley et al. (2002) portrayed that structure and adherent nature of cells in biofilms modulate the biocontrol activities and antimicrobial tolerance. $P$. putida and $P$. fluorescens bacteria are able to coat roots of plant by forming a biofilm, which may save roots against phytopathogens (Espinosa-Urgel et al., 2002; Walker et al., 2004; Ude et al., 2006). Additionally, it has been shown that besides biocontrol activities, biofilmed Pseudomonas spp. inocula possess $\mathrm{N}_{2}$-fixing properties and nutrient uptake (Seneviratne et al., 2010).

Siderophores such as pyoverdins are iron chelators molecule. Privated from iron, telluric harmful flora slows its growth and its density is decreased in the rhizosphere. This feature can enhance fluorescent Pseudomonas (PGPR) in the process of colonization and competition for the substrate better than other microbes in the rhizosphere (Lemanceau, 1992). A significant pyoverdin production was detected with isolates PsTp172, PsTp169, PsS46, PsTp171 (>80 $\mu \mathrm{M})$. Loper and Buyer (1991) explain that the pyoverdine concentration produced by Pseudomonas spp. in the rhizosphere, is important to influence the microbial interactions. Many authors found that mutants, who lost the synthesizing siderophore ability $\left(\mathrm{Sid}^{-}\right)$, are not able to determine the beneficial effects recorded with the wild strain (Becker and Cook, 1988). Unfortunately, the involvement of pyoverdin in animals and humans virulence is very well studied in pathogenic isolates $(P$. aeruginosa), but it is unlikely exposed in saprophytic strains (mainly $P$. fluorescens) grown at or above $37^{\circ} \mathrm{C}$ (Donnarumma et al., 2010). These siderophores acquire iron from lactoferrin and transferrin and regulate the production of virulence factors (exotoxin $\mathrm{A}$ and endoprotease) (Takase et al., 2000).

Besides iron, several siderophores can bind other essential metals ( $\mathrm{Mg}, \mathrm{K})$ serving as micronutrients (Shinozaki-Tajiri et al., 2004). Furthermore, microorganisms producing siderophores are able to bind a variety of toxic metals such as $\mathrm{Al}, \mathrm{Pb}, \mathrm{Cd}$ (Mureseanu et al., 2003). While essential metals have important biological role, at high levels they can alter cell membranes, disturb cellular functions, damage the DNA structure and can reduce crop yields (Teitzel et al., 2006). Pseudomonas strains are widely utilized in bioremediation processes as they are able to survive and adapt in extreme environments. PsTp172 isolate is the most tolerant bacteria to tested metals. This metal resistance could be maintained by active efflux, uptake reduction, sequestration, detoxification and binding 
proteins synthesis (Zhuang et al., 2007). In previous investigation, it has been demonstrated that PsTp171 strain was able to bind $\mathrm{Zn}$ and to tolerate high concentration of the metal. The result shows that pyoverdins may have the capacity to complex zinc instead of iron. This strain might be able to sequester one of essential element and make it unattainable to phytopathogens (competition phenomenon) (Mehri et al., 2012).

In addition to these traits, PGPB have to be competent in the rhizospheric soil (Sajani and Muthukkaruppan, 2011). So, growth inhibition of pathogenic and phytopathogenic bacteria by antimicrobials released mainly by PsS15, PsTp172, PsS28, PsTp171, PsS31, PsS67, PsS18, PsS39 and PsS93 reflect their rhizospheric competitiveness that can be beneficially combined with plant protection. On the other hand, the discovery of $Q S$ enzymes did not only offered a promising means to control bacterial infections, but also provided new challenges to study their roles in host organisms and their potential impacts on environments (Dong and Zhang, 2005). Molina et al. (2003) showed that QS signal of Erwinia carotovora and Agrobacterium tumefaciens can be disturbed by lactonase of $P$. fluorescens which significantly reduce respectively potato soft rot and crown gall of tomato. Our results revealed that PsS15, PsTp156, PsTp172, PsC54, PsTp171 and PsS102 isolates are capable to disturb and inhibit the $Q S$ signal of the 3 pathogenic and phytopathogenic bacteria. Thus, the quorum-quenching mechanism has emerged as novel biocontrol strategy that could substitute pesticides.

\section{CONCLUSION}

On the basis of biofertilizer, plant growth promoter and biocontrol capacities we propose three strains (PsTp172, PsTp171 and PsS15) as potential bioinoculants for crop plants to increase seedling emergence, vigor and yield. Selected isolates are metal tolerant and could be used in polluted soils. Further siderophore production and antagonistic activity, which helps in establishing and resisting against deleterious microorganisms, reflect their rhizospheric competitiveness and plant protection that can be beneficially combined with PGPR traits. Additionally, in vitro production of hyper-adherent and/or hyper-mucoïd PGPB inocula could be utilized to increase the crop yields by improving N2 fixation, nutrient uptake and to protect plant against soil pathogens. Moreover, the concept of biological control of plant diseases, using non-pathogenic bacteria as bio-pesticide could substitute chemical fertilizers and pesticides, since they cause an accumulation of toxic compounds in soil. Thus, the use of microorganisms as biological control agent (antimicrobial secretion or NAHL degradation) is a promising strategy to ensure effective plant protection in agricultural ecosystems.

Therefore, most effective Pseudomonas isolates could well be commercialized but we should not overlook the involvement of some species of $P$. fluorescens, able to grow at or above $37^{\circ} \mathrm{C}$ that can behave as opportunistic agent in human pathogenicity (endotoxin release). Inoculation of these species (PsS60, PsS73 and PsS91) in raw consumption plants (carrots, cabbage) can cause severe effects in patients with weakened immune systems (Chapalain et al., 2008).

\section{REFERENCES}

Barefoot, J.C., W.G. Dahlstrom and R.B. Williams, 1983. Hostility, CHD incidence and total mortality: A 25-year follow-up study of 255 physicians. Psychosom. Med., 45: 59-63. PMID: 6844529

Becker, J.O. and R.J. Cook, 1988. Role of siderophores in suppression of pythium species and production of increased-growth response of wheat by fluorescent pseudomonads. Phytopathology, 78: 778-782. DOI: 10.1094/Phyto-78-778

Bianciotto, V., S. Andreotti, R. Balestrini, P. Bonfante and S. Perotto, 2001. Mucoid mutants of the biocontrol strain Pseudomonas fluorescens CHA0 show increased ability in biofilm formation on mycorrhizal and nonmycorrhizal carrot roots. Mol. Plant-Microbe Interact., 14: 255-60. PMID: 11204790

Bloemberg, G.V. and B.J.J. Lugtenberg, 2001. Molecular basis of plant growth promotion and biocontrol by rhizobacteria. Curr. Opinion. Plant. Biol., 4: 343350. DOI: 10.1016/S1369-5266(00)00183-7

Chapalain, A., G. Rossignol, O. Lesouhaitier, A. Merieau and C. Gruffaz, 2008. Comparative study of 7 fluorescent pseudomonad clinical isolates. Rev. Can. Microbiol., 54: 19-27. DOI: 10.1139/W07-110

Compant, S., B. Duffy, J. Nowak, C. Clément and E. Ait Barka, 2005. Use of plant growth-promoting bacteria for biocontrol of plant diseases: Principles, mechanisms of action and future prospects. Appl. Environ. Microbiol., 7: 94951-4959. PMID: 16151072

Danhorn, T. and C. Fuqua, 2007. Biofilm Formation by Plant-Associated Bacteria. Annu. Rev. Microbiol., 61: 401-422. DOI: 10.1146/annurev.micro.61.080706.093316 
Dong, Y.H. and L.H. Zhang, 2005. Quorum sensing and quorum-quenching enzymes. J. Microbiol., 43: 101-109. PMID: 15765063

Donnarumma, G., E. Buommino, A. Fusco, I. Paoletti and L. Auricchio et al., 2010. Effect of temperature on the shift of Pseudomonas fluorescens from an environmental microorganism to a potential human pathogen. Int. J. Immunopathol. Pharmacol., 23: 227-234. PMID: 20378008

Espinosa-Urgel, M., R. Kolter and J.L. Ramos, 2002. Root colonization by Pseudomonas putida: Love at first sight. Microbiology, 148: 341-343. PMID: 11832496

Freeman, D.J., F.R. Falkiner and C.T. Keane, 1989. New method for detecting slime production by coagulase negative staphylococci. J. Clin. Pathol., 42: 872-874.

Gordon, S.A. and R.P. Weber, 1951. Colorimetric estimation of indole-acetic acid. Plant. Physiol., 26: 192-195. DOI: $10.1104 /$ pp.26.1.192

Husen, E., A.T. Wahyudi, A. Suwanto and Giyanto, 2011. Soybean Response to 1-Aminocyclopropane-1Carboxylate Deaminase-Producing Pseudomonas under Field Soil Conditions. Am. J. Agri. Biol. Sci., 6: 273-278. DOI: 10.3844/ajabssp.2011.273.278

Katznelson, H. and B. Bose, 1959. Metabolic activity and phosphate-dissolving capability of bacterial isolates from wheat roots, rhizosphere and nonrhizosphere soil. Can. J. Microbiol., 5: 79-85. DOI: 10.1139/m59-010

King, E.O., M.K. Ward and D.E. Raney, 1954. Two simple media for the demonstration of pyocyanin and fluorescin. J. Lab. Clin. Med., 44: 301-307. PMID: 13184240

Kirisits, M.J., L. Prost, M. Starkey and M.R. Parsek, 2005. characterization of colony morphology variants isolated from pseudomonas aeruginosa biofilms. Appl. Environ. Microbiol., 71: 4809-4821. DOI: 10.1128/AEM.71.8.4809-4821.2005

Kumar, A., A. Kumar, S. Devi, S. Patil, C. Payal and S. Negi, 2012. Isolation, screening and characterization of bacteria from Rhizospheric soils for different Plant Growth Promotion (PGP) activities: An in vitro study. Recent Res. Sci. Technol., 4: 01-05. DOI: $10.1007 / \mathrm{s} 10725-012-9670-\mathrm{x}$

Lemanceau, P., 1992. Effets bénéfiques de rhizobactéries sur les plantes: Exemple des Pseudomonas spp fluorescents. Agronomie, 12: 413-437. DOI: 10.1051/agro:19920601

Loper, J.E. and J.S. Buyer, 1991. Siderophores in microbial interactions on plant surfaces. Curr. Rev., 4: 5-13. DOI: 10.1094/MPMI-4-005
Mehri, I., A. Khessairi, Y. Turki, N. Saidi, I. Daly, 2012. Dose-response effect of metals on siderophores production produced by environmental Pseudomonads. Am. J. Environ. Sci., 8: 143-151. DOI: 10.3844/ajessp.2012.143.151

Mehri, I., Y. Turki, M. Chair, H. Chérif, A. Hassen, J.M. Meyer and M. Gtari, 2011. Genetic functional heterogeneities among fluorescent Pseudomonas isolated from environmental samples. J. Gen. Applic. Microbiol., 57:101-114. DOI:10.2323/jgam.57.101

Meyer, J.M. and M.A. Abdallah, 1978. The fluorescent pigment of Pseudomonas fluorescens: Biosynthesis, purification and physiocochemical properties. J. Gen. Microbiol., 107: 319-328. DOI: 10.1099/00221287-107-2-319

Mishra, M., P., Nandy, S. Debsarma, M. Dutta and A.K. Sarkar et al., 2010.Condoms-As Sources of Extracellular Enzyme Producing Microbes. Am. J. Microbiol. 1: 14-22. DOI: 10.3844/ajmsp.2010.14.22

Molina, L., F. Constantinescu, L. Michel, C. Reimmann, B. Duffy and G. Défago, 2003. Degradation of pathogen quorum sensing molecules by soil bacteria: A preventive and curative biological control mechanism. FEMS Microbiol. Ecol., 45: 7181. DOI: 10.1016/S0168-6496(03)00125-9

Munsch, P., V. Geoffroy, T. Alatossava and J.M. Meyer, 2000. Application of Siderotyping for Characterization of Pseudomonas tolaasii and "Pseudomonas reactans" Isolates Associated with brown blotch disease of cultivated mushrooms. Applic. Environ. Microbiol., 6: 4834-4841. DOI: 10.1128/AEM.

Mureseanu, M., G. Renard, A. Galarneau and D.A. Lerner, 2003. A demonstration model for a selective and recyclable uptake of metals from water: Fe (III) ions complexation and release by a supported natural fluorescent chelator. Talanta, 60: 515-522. DOI: $10.1016 / S 0039-9140(03) 00103-6$

O'Toole, G.A., L.A. Pratt, P.I. Watnick, D.K. Newman and V.B. Weaver et al., 1999. Genetic approaches to the study of biofilms. Methods Enzymol., 310: 91109. DOI: 10.1016/S0076-6879(99)10008-9

Ouzari, H., A. Khsairi, N. Raddadi, L. Jaoua, A. Hassen, 2008. Diversity of auxin-producing bacteria associated to Pseudomonas savastanoi-induced olive knots. J. Basic. Microbiol., 48: 18. DOI:10.1002/jobm. 200800036

Patten, C.L. and B.R. Glick, 2002. Regulation of indoleacetic acid production in Pseudomonas putida GR12-2 by tryptophan and the stationary phase sigma factor RpoS. Can. J. Microbiol., 48: 635-642. DOI: $10.1139 / w 02-053$ 
Pradhan, N. and L.B. Sukla, 2006. Solubilization of inorganic phosphates by fungi isolated from agriculture soil. Afr. J. Biotechnol., 5: 850-854.

Rainey, P.B. and M. Travisano, 1998. Adaptive radiation in a heterogeneous environment. Nature, 394: 69-72. DOI: $10.1038 / 27900$

Rakhimova, E., A. Munder, L. Wiehlmann, F. Bredenbruch and B. Tümmler, 2008. Fitness of isogenic colony morphology variants of Pseudomonas aeruginosa in Murine Airway infection. PLos One, 3: 1-16. DOI: 10.1371/journal.pone.0001685

Rodriguez, H. and R. Fraga, 1999. Phosphate solubilizing bacteria and their role in plant growth promotion. Biotechnol. Adv., 17: 319-339. DOI: 10.1016/S0734-9750(99)00014-2

Saharan, B.S. and V. Nehra, 2011. Plant growth promoting rhizobacteria: A critical review. Life Sci. Med. Res.

Sajani, S. and S.M. Muthukkaruppan, 2011. Characterization of plant growth promoting rhizobacteria and fungi associated with rice, mangrove and effluent contaminated soil. Curr. Bot., 2: 22-25.

Seneviratne, G., M.L.M.A.W. Weerasekara, K.A.C.N. Seneviratne, J.S. Zavahir and M.L. Kecske's et al., 2010. Importance of Biofilm Formation in Plant Growth Promoting Rhizobacterial Action. In: Plant Growth and Health Promoting Bacteria, Microbiology Monographs 18, Mahrshwari, D.K., (Eds.), Springer-Verlag Berlin Heideberg, pp: 81-69. DOI: $10.1007 / 978-3-642-13612-2 \_4$

Shinozaki-Tajiri, Y., Y. Akutsu-Shigeno, T. NakajimaKambe, S. Inomata and N. Nomura et al., 2004. Matrix metalloproteinase-2 inhibition and $\mathrm{Zn}^{2+}$ chelating activities of pyoverdine-type siderophores. J. Biosci. Bioeng., 97: 281-283. DOI: 10.1016/S1389-1723(04)70205-4

Stoodley, P., K. Sauer, D.G. Davies and J.W. Costerton, 2002. Biofilms as complex differentiated communities. Annu. Rev. Microbiol., 56: 187-209. DOI: 10.1146/annurev.micro.56.012302.160705

Swift, S., P. Williams and G.S.A.B. Stewart, 1999. Quorum sensing in Aeromonas and Yersinia. In: Microbial Signalling and Communication, England, R., G. Hobbs, N.J. Bainton and D.M. Roberts (Eds.), Cambridge University Press, Cambridge, UK., pp: 85-104.
Takase, H., H. Nitanai, K. Hoshino and T. Otani, 2000. Impact of siderophore production on Pseudomonas aeruginosa infections in immunosuppressed mice. Infect. Immun. 68: 1834-1839. DOI: 10.1128/IAI.68.4.1834-1839.2000

Teitzel, G.M., A. Geddie, S.K. DeLong, M.J. Kirisits, M. Whiteley and M.R. Parsek, 2006. Survival and growth in the presence of elevated copper: Transcriptional profiling of copper-stressed Pseudomonas aeruginosa. J. Bacteriol., 188: 72427256. DOI: $10.1128 /$ JB.00837-06

Ude, S., D.L. Arnold, C.D. Moon, T. Timms-Wilson and A.J. Spiers, 2006. Biofilm formation and cellulose expression among diverse environmental Pseudomonas isolates. Environ. Microbiol., 8: 19972011. DOI: 10.1111/j.1462-2920.2006.01080.x

Villegas, J. and J.A. Fortin, 2002. Phosphorus solubilization and $\mathrm{pH}$ changes as a result of the interactions between soil bacteria and arbuscular mycorrhizal fungi on a medium containing $\mathrm{NO}_{3}{ }^{-}$as nitrogen source. Can. J. Bot., 80: 571-576. DOI: 10.1139/b02-038

Von Bodman, S.B., W.D. Bauer and D.L. Coplin, 2003. Quorum sensing in plant-pathogenic bacteria. Annu. Rev. Phytopathol., 41: 455-482. DOI: 10.1146/annurev.phyto.41.052002.095652

Wahyudi, A.T., R.I. Astuti and Giyanto, 2011. Screening of Pseudomonas sp. Isolated from rhizosphere of soybean plant as plant growth promoter and biocontrol agent. Am. J. Agri. Biol. Sci., 6: 134-141. DOI: 10.3844/ajabssp.2011.134.141

Walker, T.S., H.P. Bais, E. Deziel, H.P. Schweizer, L.G. Rahme et al., 2004. Psedomonas aeruginosa plant root interactions. Pathogenicity, biofilm formation and root exudation. Plant Physiol., 134: 320-331. DOI: $10.1104 /$ pp.103.027888

Zhuang, X., J. Chen, H. Shim and Z. Bai, 2007. New advances in plant growth-promoting rhizobacteria for remediation. Environ. Int., 33: 406. DOI: 10.1016/j.envint.2006.12.005 